\title{
Site Accessibility Prediction with Random Forest Algorithm and Quadruped Bot
}

\author{
Akshaymon KV ${ }^{1}$, Arjun Anil ${ }^{2}$, Ashish Philip ${ }^{3}$, Gladstan K Saji ${ }^{4}$, Sujitha $\mathrm{M}^{\mathbf{5}}$ \\ ${ }^{1}$ Mangalam College of Engineering, Kottayam, India, kvakshaymon@ gmail.com \\ ${ }^{2}$ Mangalam College of Engineering, Kottayam, India, arjun1anil@ gmail.com \\ ${ }^{3}$ Mangalam College of Engineering, Kottayam, India, ashishphilip1001@ gmail.com \\ ${ }^{4}$ Mangalam College of Engineering, Kottayam, India, gladstankizhakkel@ gmail.com \\ ${ }^{5}$ Mangalam College of Engineering, Kottayam, India, m.sujitha@mangalam.in
}

\begin{abstract}
Quadruped robots are legged robots that can adapt to various structural locomotions. Legged robots are mostly used for surveillance and stealth purposes in military and disaster management [1]. They are rarely used in day to day life. This paper introduces a way to bring about the use of quadruped bot into day to day life. Such a bot can be used to attach several sensors to collect surrounding environmental data and this data can be used to predict whether a particular place or area is safe to be approached by a certain group of people. The grouping based on age group and health conditions.
\end{abstract}

Key words: Random Forest algorithm, Arduino microcontroller, inverse kinematics, thingspeak cloud, Iot, neural network.

\section{INTRODUCTION}

Atmospheric conditions are constantly changing nowadays due to climate change and increased pollution. This change takes place randomly at different places and hence it is not the same everywhere. Therefore all the places are not safely accessible by everyone. Air quality ,pressure and temperature are some of the key factors to be considered when measuring the safety of a place. Constant implant of sensors everywhere is costly and not practical. Hence the person should be able to have something that can be carried around.

A legged robot can be used for this purpose since they can move easily on uneven terrains when compared with the widely used wheeled robots. Most of earth's landscape is uneven and not accessible to wheeled equipment. The user can direct the bot to travel into the intended place and collect all the data to predict where the place is accessible to him/her or anyone needed to access the place. The bot used in the project is a Quadruped. It is a four legged robot with the body framework similar to a spider. It also adapts the frameworks's locomotion An arduino microcontroller with a custom made PCB is used to embedded the needed sensors [2].

\section{RELATED WORKS}

Quadruped robots or generally legged robots are used for surveillance and stealth operations since they can be controlled through a mobile application from a desired distance based on the range of the system used. Boston dynamics are the industry leading company in quadruped bot research. They have demonstrated the use of such robots in inspecting construction and excavation sites so that they can be commercially used. A large number of small scale quadruped bots are available and are built using custom made pcb's. Microcontrollers such as Arduino, Raspberry etc are used.

For measuring weather and atmospheric conditions at large scale the reliable options are weather stations and google's vertical profile measurements. The problem with them is they cannot be localised to a small particular area.

\section{BASIC COMPONENTS}

The system proposed can be divided into four different functional parts ard are listed below respectively:

\subsection{QUADRUPED STRUCTURE}

The quadbot structure is made up of laser cut acrylic ram material designed in coreldraw [1]. The structure consists of a flat base and leg structures, 8 SG90 
servo motors are fitted into these leg structures where the joint happens. The servo motors are connected to the ATMEGA microcontroller. Inverse kinematics is used to angle the servos for locomotion. Electronic signal jammers and laser diodes are toggled into the system. A series of sensors used to collect data are embedded into the body frame. Other sensors that are embedded into the board that are controlled by the ATMEGA microcontrollers are listed in the below table.

Table 1: Sensors Used

\begin{tabular}{|l|l|}
\hline Arduino Mega & $\begin{array}{l}\text { Microcontroller } \\
\text { ATMEGA } \\
\text { microcontrollers are listed } \\
\text { in the below table. }\end{array}$ \\
\hline KY-038 & Sound sensor \\
\hline BMP-180 & BMP sensor \\
\hline DHT22 & Temperature \& Humidity \\
\hline LDR & Light Sensor \\
\hline RSM & Soil moisture \\
\hline LM393 & Rain sensor \\
\hline HC-SR04 & UltraSonic Sensor \\
\hline KY-008 & Laser \\
\hline ESP-8266 & WiFi Module \\
\hline HC-05 & Bluetooth Module \\
\hline
\end{tabular}

\subsection{ARDUINO MICROCONTROLLER}

The Arduino Mega is a microcontroller board based on the ATmega2560 (datasheet). It has 54 digital input/output pins (of which 14 can be used as PWM outputs), 16 analog inputs, 4 UARTs (hardware serial ports), a $16 \mathrm{MHz}$ crystal oscillator, a USB connection, a power jack, an ICSP header, and a reset button. It contains everything needed to support the microcontroller [9]. The microcontroller is used to program the sensors to collect data.

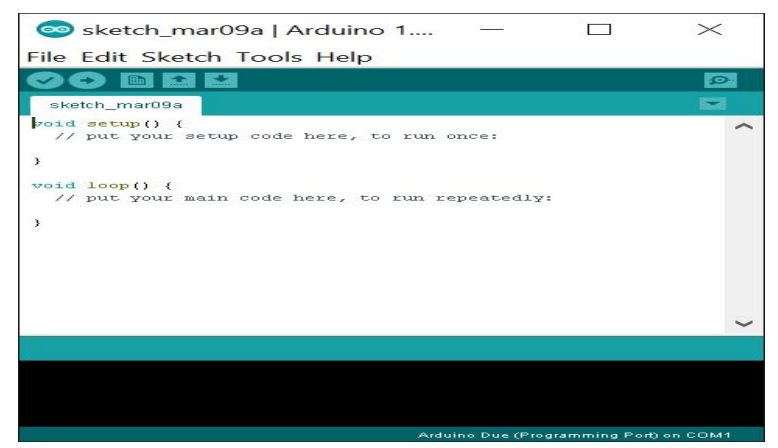

Figure 1: Arduino IDE

\section{3 MOBILE APPLICATION}

The movement of the Quadruped robot is controlled using a third party application ArduSim. It provides the SDK tools to control the arduino used. The servos used for locomotion are controlled by the microcontroller along with the other sensors. The HC-05 Bluetooth module on the Quadruped Bot is used for establishing connection with ArduSim. Once the connection has been established the controls on the user interface of ArduSim allows us to control the movement of Bot. It also consists of an IP cam embedded on the body for surveillance and can be used to control the bot from distances.

\subsection{PREDICTION SYSTEM}

The sensors that are listed above collect the atmospheric and environmental data of the place they are driven into and this data is in sync with the cloud. The cloud system used in the proposed system is Thingspeak. The data that is collected by the cloud is forwarded to a prediction algorithm to generate the desired prediction.

\section{PROPOSED SYSTEM}

The proposed system uses a basic quadruped structure made up of acrylic to avoid radar incase if it needs to be used for surveillance. Two Servo SG90 motors are used in each leg which are programmed for locomotion. Initial angle and angle of rotation at each leg position is mapped based on the inverse kinematics equation. The servo motors are connected to the PWM pins of an ATmega 2560 microcontroller An additional sensor array consisting of both digital and analog ones are also connected to the controller using a sensor shield. These sensor reading are analysed within the controller for making decisions about the movements and data is later pushed into the thingspeak cloud 


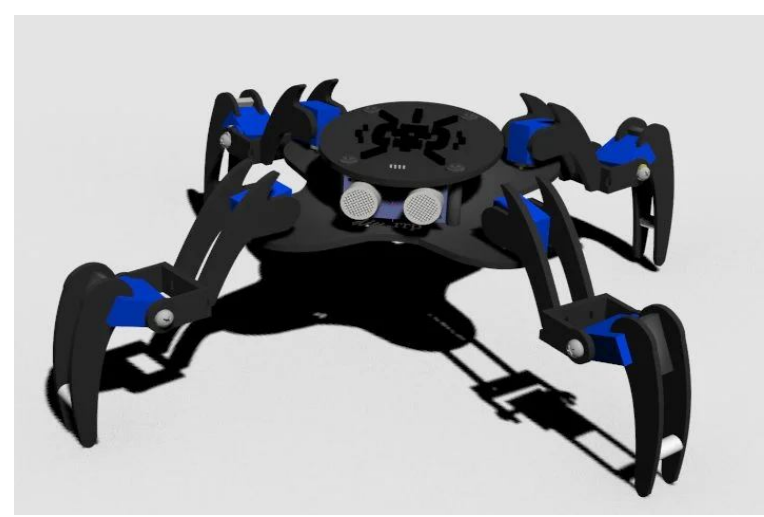

Figure 2: Quadruped robot structure [10]

Arduino IDE is used to upload the code to Mega [7]. The sensors are programmed using embedded C. Seperate programs are used for each sensor for data collection [8].For controlling purposes, HC05 bluetooth module and ESP 8266 of 802.11 standard is used. IP cams are used for further vision assistance to the person who controls the quadbot.

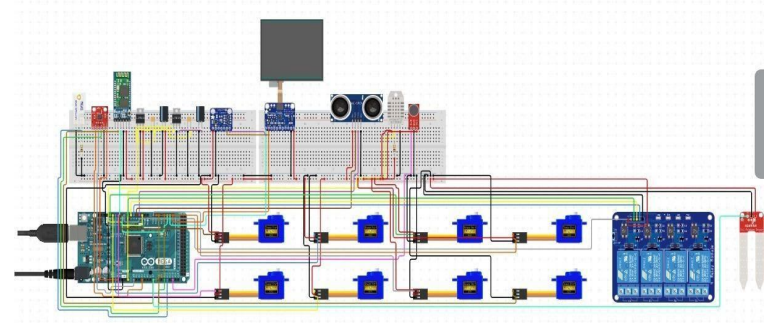

Figure 3: Proposed system circuit diagram

\subsection{SYSTEM ARCHITECTURE}

The system proposed is data collection for which we use different sensors. The quadruped robot is deployed by the user into the desired area or place to be checked for safety. The bot is controlled through the ArduSim application with the help of Iocam embedded in the framework. All the sensor data collection is programmed using embedded $\mathrm{C}$ in the arduino IDE. The DHT22 sensor reads the temperature and humidity of the area. Rain drop detection sensor constantly checks for the presence of rain. BMP-180 sensor reads the pressure and altitude of the area. Light sensor checks for any presence of light, Sound sensor constantly monitors the sounds around the area. Similarly each of the tables that are listed in table [1], collects the data that are intended by them and sends them to the Thingspeak cloud. In case of non availability of Internet connection an SD card module is planted along with the sensors and hence ensures that the data is not lost.All these sensor readings are sent to thingspeak cloud via api pin and ESP-8266 WiFi module.
Second phase of the system proposed is prediction using the collected data. The data that is collected in the cloud is converted into CSV format. The collected data is used to predict what group of people can visit a particular place. Then, the data is fed into the AI model in the form of CSV files. Random Forest Algorithm is used inorder to build the AI model. It is implemented using Python IDLE.

Random Forest Algorithm is used inorder to build the AI model. The classification of type or group of people is based on the respective age group to which they belong.

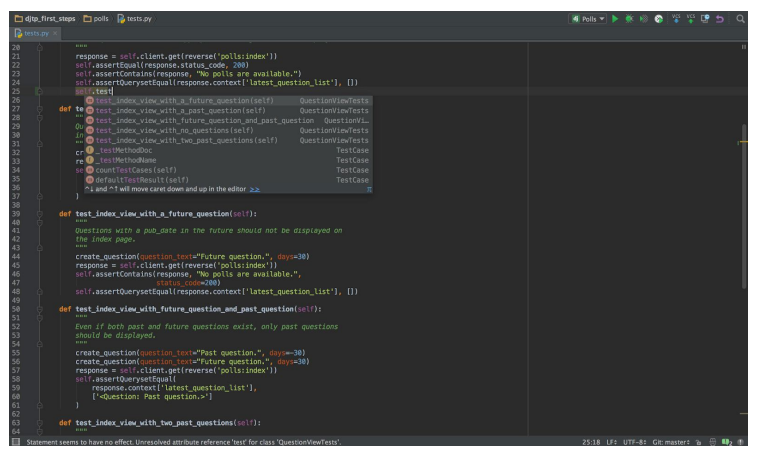

Figure 4: Python IDLE

Table 2: Dataset provided to AI model

\begin{tabular}{|c|c|c|c|c|c|}
\hline Air Quality & Humidity & Temperature & Sound & Light & Safe Popuation \\
\hline $30^{4}$ & 35 & 16 & 30 & & Suitable for Babies \\
\hline 120 & 80 & 35 & 50 & & Not Suitable For Babies \\
\hline 50 & 30 & 20 & 40 & & Suitable for kids under 10 \\
\hline 51 & 33 & 22 & 50 & & Suitable for children under 18 \\
\hline 60 & 37 & 36 & 69 & & Suitable for aduts \\
\hline 55 & 42 & 34 & 70 & & Not suitable for people with heart diseases \\
\hline 90 & 38 & 37 & 90 & & Moderately suitable for adults \\
\hline 310 & 45 & 40 & 140 & & Hazardous to humans \\
\hline 420 & 50 & 45 & 120 & & Hazardous to humans \\
\hline 32 & 31 & 20 & 50 & & Suitable for babies \\
\hline 35 & 55 & 40 & 57 & & Not suitable for babios \\
\hline 37 & 33 & 30 & 40 & & Suitable for kids under 10 \\
\hline 40 & 50 & 37 & 75 & & Suitable for aduts \\
\hline 42 & 32 & 35 & 60 & & Suitable for children above 18 \\
\hline 43 & 55 & 35 & 60 & & Not suitable for chidren under 18 \\
\hline 31 & 35 & 40 & 90 & & Suitable for aduts \\
\hline 35 & 75 & 60 & 50 & & Not suitable for aduts \\
\hline 46 & 70 & 60 & 140 & & Not suitable for aduts \\
\hline 40 & 40 & 24 & 65 & & Suitable fo Asthma Patients \\
\hline 290 & 70 & -12 & 130 & & Not Suitable for Asthma Patients \\
\hline
\end{tabular}

Algorithm

Step 1: First, start with the selection of random samples from a given dataset.

Step 2: Next, this algorithm will construct a decision tree for every sample. Then it will get the prediction result from every decision tree.

Step 3: In this step, voting will be performed for every predicted result. 
Step 4: At last, select the most voted prediction result as the final prediction result.

Inorder to train the model initially a minimal dataset of 100 rows and 6 columns with predetermined sensor values are used. The algorithm is programmed in Python IDLE which provides the desirable efficiency and accuracy required for the prediction model.

\section{RANDOM FOREST ALGORITHM}

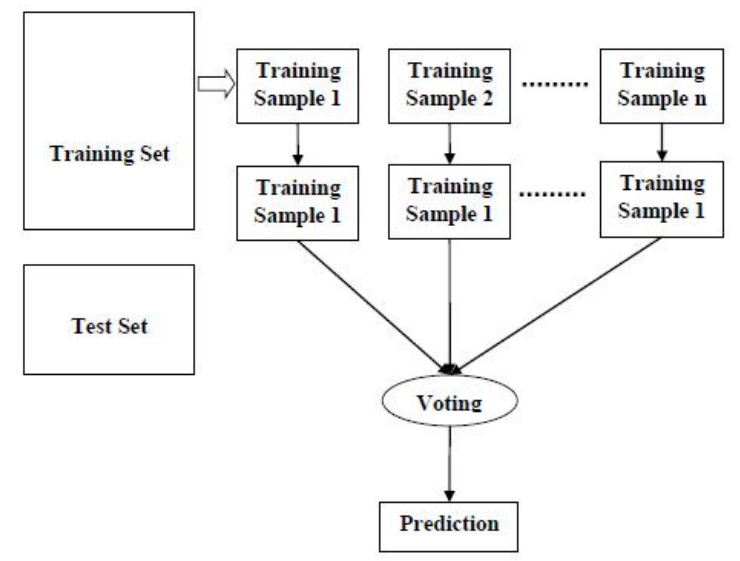

Figure 5: Working of the RF Algorithm

Random forest algorithm uses supervised learning techniques which can be used for both regression and classification. Like the learning technique mentioned, a random forest algorithm makes decision trees based on the provided data samples and then gets the prediction from each of the decision trees generated and by carrying out voting to obtain the best solution [11].

\section{ADVANTAGES}

1. It overcomes the overfitting problem by combining all the obtained decision tree results.

2. The algorithm works for a very large amount of data.

3. The variance of random forest is less than a single decision tree.

4. Really flexible and accurate.

\section{DISADVANTAGES}

One of the most important disadvantages of random forest algorithms is it's complexity. It is really harder to construct random first and is less intuitive when there is a large number of decision trees.

\section{RESULT}

The data collected from the bot is stored in the thingspeak cloud and this data is forwarded to the algorithm to identify whether the particular atmospheric and environmental condition is suitable for a certain group or type of people classified based on their age group and health condition.

\section{CONCLUSION}

Wheeled robots being not utilisable in uneven terrains the structure proposed is a quadruped with spider's locomotion.The system proposes a way in which surveillance bots can be used by common people in identifying if certain places are accessible to the associated type of people. People can be categorized based on their age group or health conditions.

The system uses an Arduino microcontroller to attach the sensors required to collect atmospheric and other required readings and values to identify if the place is accessible. Once the data is collected it is uploaded to a cloud server.The data collected is forwarded to the random forest algorithm and the algorithm determines the result. Once the result is obtained it is forwarded to the user.

The bot being controlled using a mobile application simplifies its usability. The bot can be used for a variety of purposes alongside surveillance and the proposed prediction. This can also be considered to be the future scope of the project as it can be used by police and fire force in disaster management and prediction. The proposed project will be built on ATmega 2560 microcontroller and its allied components. Embedded C codes will be used to programme the ATmega controller via the Arduino IDE for the locomotion of proposed quadbot and can also be used for retrieving data and sending it to thingspeak cloud.

\section{ACKNOWLEDGMENT}

The authors thank Principal Manoj George, Dr. Vinod P Vijayan, H.O.D, Department of Computer Science, Mrs. Sujitha M for the proper guidance, valuable support and helpful comments during the proofreading. 


\section{REFERENCES}

[1] Yam Geva, Amir Shapiro : A Novel Design of a Quadruped Robot for Research Purposes, January 1,2014

[2] MM Gori, P M Pathak, A K Samantaray : Development of a Compliant Legged Quadruped Robot, 18 June 2018

[3] T.T.K Dat,Phuc Thien Tran : A Study on Locomotions of Quadruped Robot, Ho Chi Minh City University of Technology, Vietnam, 2013

[4] Sachin Oak, Vaibhav Narwane : Design and Fabrication of Quadruped Robot with Four bar Chain Leg Mechanism, 2014

[5] Byeong Hun Na, Kyoungchul Kong : Design of a One Degree-of-Freedom Quadruped Robot Based on a Mechanical Link System, 2016

[6] https://www.instructables.com/id/2dof

Quadruped-Robot-With-Acrylic-Laser-Cut-Frame/

[7] https://blog.arduino.cc/

[8]https://learn.adafruit.com/adafruit-all-aboutarduino-libraries-install-use

[9] https://github.com/esp8266/Arduino

[10]https://images.google.com/imghp?hl=en\&gl=ar\& gws_rd=ssl

[11] Effective Learning and Classification using Random Forest Algorithm Vrushali Y Kulkarni, Pradeep K Sinha 